\title{
Outcome of pregnancy following IVF/ IUI complicated by ovarian torsion: case reports
}

\section{Garima Sachdeva, Shalini Gainder*}

\begin{abstract}
Department of Obstetrics and Gynecology, Post Graduate Institute of Medical Education and Research, Chandigarh,
\end{abstract} Punjab, India

Received: 25 September 2018

Accepted: 22 October 2018

\author{
*Correspondence: \\ Dr. Shalini Gainder, \\ E-mail: shalini_gainder@yahoo.com
}

Copyright: (c) the author(s), publisher and licensee Medip Academy. This is an open-access article distributed under the terms of the Creative Commons Attribution Non-Commercial License, which permits unrestricted non-commercial use, distribution, and reproduction in any medium, provided the original work is properly cited.

\begin{abstract}
Ovarian torsion though uncommon after in vitro fertilization (IVF)/ intrauterine insemination (IUI), but if not diagnosed early can lead to ischemic necrosis of the ovary. The reported incidence of ovarian torsion after IVF/IUI is $0.025-0.2 \%$ and is primarily attributed to controlled ovarian stimulation (COS). Here we present three case reports of ovarian torsion after IVF/IUI reported in a government hospital, India. The first case was referred to our institute after IVF with acute onset abdominal pain. Diagnosis of ovarian torsion was made. In this patient, ovaries could be salvaged by early diagnosis and intervention and she even delivered a $2.95 \mathrm{~kg}$ girl baby in the same pregnancy. The second case also followed IVF done in our institute and by early diagnosis and intervention, we could salvage the ovary. The third case followed IUI, but due to delayed presentation, the patient had to undergo ipsilateral salpingooopherectomy. A total of 1562 IUI and 98 IVF were done at our institute last year, of which only one case each of IUI and IVF landed up in ovarian torsion. The significance of this article is to discuss the diagnosis and management of ovarian torsion and to reiterate the importance of early diagnosis and management.
\end{abstract}

Keywords: Human chorionic gonadotropin In vitro fertilisation, Intrauterine insemination, Ovarian stimulation, Torsion

\section{INTRODUCTION}

Often, women who wish to conceive, seek rescue in IVF/IUI treatments. However, the disappointing fact is that sometimes due to ovarian torsion they might end up losing their ovaries. The estimated incidence of ovarian torsion after in vitro fertilization (IVF)/ in utero insemination (IUI) is $0.025-0.2 \%{ }^{1}$ With increasing incidence of infertility, the need for IVF/IUI is also increasing. It is a common practice to do controlled ovarian stimulation (COS) before IVF/IUI. ${ }^{2}$ As a result of superovulation, there may be a rise in pregnancy rates. This however, also increases the risk of ovarian hyperstimulation syndrome (OHSS) and hence ovarian torsion. ${ }^{3}$ Also, the persistent secretion of human chorionic gonadotrophin (HCG) by the placenta and ovary, also adds to the risk of ovarian torsion. ${ }^{4}$ Ovarian torsion is a gynecological emergency. ${ }^{5}$ Any kind of delay in diagnosis or management will lead to permanent ischemic necrosis of the ovaries. Early intervention can improve outcome both in the mother and fetus. So, in all cases of acute abdominal pain in early pregnancy after IVF/IUI treatment, ovarian torsion should be ruled out. Here we report our experience in managing ovarian torsion at our institute.

\section{CASE REPORT}

\section{Case 1}

A 29-year-old presented to a clinic at a private hospital with secondary infertility for 3 years. She had a history of previous one first trimester loss and was a known case of the polycystic ovarian syndrome (PCOS) for 5 years and 
hypothyroidism for 3 years. History of male factor infertility was also present (husband had a history of varicocele 2 years back and he had a sperm count of 5 million/ml). She underwent IVF (in vitro fertilization) at the private hospital after which she conceived. On the 8th day post conception, she developed abdominal pain, nausea, vomiting. Bilateral pleural effusion and ascites were present. She was managed conservatively with bilateral pleurocentesis. At 6 weeks 5-day period of gestation (POG) her ultrasonography (USG) revealed bilaterally enlarged ovaries with multiple cysts, few with hemorrhage. Diagnosis of ovarian hyperstimulation syndrome (OHSS) with cystic hemorrhage was made. Conservative management was done with bilateral pleurocentesis (around $300 \mathrm{ml}$ fluid aspirated). At 9-week 3 days POG, her USG revealed triplet gestation with two live fetuses of 9 weeks and 8 weeks 6 days' size respectively and a fetus of 7 -week size with no cardiac activity. At 13 weeks 3 days POG, the patient developed complain of sudden onset pain abdomen and for the first time presented in our institute. On examination, she was afebrile, her pulse rate was $92 / \mathrm{min}$, blood pressure of $110 / 70$. Her abdomen was soft with tenderness present in the left lower abdomen. Her blood investigations, CXR (Chest-X-Ray), and ECG (Electrocardiogram) were within normal limits. Her USG at 13 weeks 3 days POG, showed the presence of twin pregnancy of 14 weeks 4 days and 12 weeks 5 days' size respectively. Twin 1 had the heart rate of $125 \mathrm{bpm}$ and twin 2 did not show any cardiac activity. Inter-twin membrane was thin (3 mm). A third compartment was also seen with no fetal pole within it. The left adnexa measured $10.8 \times 9 \times 8 \mathrm{~cm}$ with multiple cysts largest measuring $6 \times 7.5 \mathrm{~cm}$ with one of the cysts showing echogenic peripheral content probably area of hemorrhage. The right adnexa measured $8 \times 7.5$ $\mathrm{X} 7 \mathrm{~cm}$ with multiple cysts. There was no evidence of free fluid. Liver, spleen, pancreas, bilateral kidneys, and gall bladder were normal. There was no evidence of torsion. The patient was given injection of hydroxyprogesterone caproate and intravenous paracetamol but, continued to have pain with tenderness in the left lower abdomen and decision for laparotomy was made. The abdomen was opened with a midline infraumblical incision. The uterus was 18 weeks' size. Left ovary revealed two coils of torsion. Detorsion was done. Multiple cysts were present in both ovaries, largest 9 X $6 \mathrm{~cm}$, same removed and specimen sent for histopathology. Histopathology revealed hemorrhagic and corpus luteal cyst in left ovary and benign cyst in the right ovary. Postoperative vitals were stable, and the patient was discharged after 3 days of observation in a stable condition on weekly progesterone. Following this the pregnancy was uncomplicated and delivered a live born girl, $2.953 \mathrm{~kg}$ at 38 weeks 5 days POG.

\section{Case 2}

A 34-year-old female had a history of primary infertility for 5 years. During her evaluation for infertility hysterolaproscopy was done in a private hospital, Patna, in which uterine cavity was normal and bilateral tubes had a loculated appearance. Tb PCR of omental biopsy was positive. She received anti-tubercular treatment for 6 months following which Endometrial biopsy was negative for tuberculosis. After multiple failed cycles of ovulation induction and 6 cycles of IUI, plan for IVF was made. Ovum pick followed by embryo transfer was done. The patient was apparently well until 5 days after embryo transfer, when she developed complains of acute onset severe pain in lower abdomen on the right side. There was no history suggestive of nausea, vomiting, decreased urine output or shortness of breath. Her hemoglobin was $9 \mathrm{gm} \%$ and her RFT, LFT, ECG and, CXR were within normal limits. Her USG revealed that right ovary was enlarged $(11.5 \times 7 \times 8.3 \mathrm{~cm}, 360 \mathrm{cc})$ in size as compared to left ovary $(6.2 \times 5.6$ X $5 \mathrm{~cm}, 100 \mathrm{cc})$. Multiple follicles were present in bilateral ovaries. Moderate free fluid was present in the pouch of Douglas. Doppler revealed decreased vascularity in right ovary. The decision for urgent laparoscopy made. On laparoscopy, hemoperitoneum was present $(100 \mathrm{cc})$. Left ovary was enlarged, normal looking while right ovary was enlarged and hemorrhagic looking. A decision for laparotomy made in view of the difficulty in laparoscopy as ovaries were very bulky and enlarged. Abdomen opened by a midline infraumblical incision. Right ovary was hemorrhagic looking while its base was normal looking. Two coils of torsion were present at the base of right ovary. Left ovary and bilateral tubes were normal looking. Right ovary was detwisted. Hemorrhagic area of right ovary was removed and sent for histopathology followed by reconstruction of the right ovary. The patient was observed for 3 days and discharged under stable condition.

\section{Case 3}

A 32-year-old female had a history of primary infertility for 5 years. On evaluation, her bilateral ovaries were enlarged and polycystic. Her prolactin and TSH levels were within normal limits. Husband semen analysis and hysterosalpingography report were within normal limits. Her endometrial biopsy revealed hyperplasia with atypia. She received progesterone treatment for 1 year and repeat endometrial biopsy had no evidence of hyperplasia. She received clomiphene citrate treatment for 1 year but due to its failure, a decision was made for intrauterine insemination. She conceived after her first cycle of IUI. During this period, she had mild pain abdomen on and off with no evidence of abdominal distention, vomiting, and shortness of breath. She was managed conservatively on the terms of mild OHSS. At $8+4$ weeks POG, her abdominal pain increased in intensity and she presented to the emergency department. There was no history of nausea, vomiting, shortness of breath or decreased urine output. Her USG revealed a single live intrauterine fetus. Right ovary was enlarged $(10 \times 8 \times 7 \mathrm{~cm})$ as compared to left $(3 \times 2 \times 2 \mathrm{~cm})$. Multiple small cysts were present in the periphery in bilateral ovaries. Mild ascites was present. Her hemoglobin was $9.8 \mathrm{gm} \%$ and her LFT, 
RFT, and urine output were within normal limits. Her pain was not subsiding on routine analgesics. Repeat USG Doppler was done which revealed enlarged right ovary $(12.9 \times 7.5 \mathrm{~cm})$ with no sign of vascularity (arterial or venous) within right ovary suggestive of torsion. The left ovary was normal. An immediate decision of laparotomy was made. The abdomen was opened with a midline infraumblical incision. Right ovary was twisted twice around its pedicle, same was detwisted. The whole of the tubo-ovarian mass was devitalized. Right-sided salpingo-oopherectomy was done. No nodularity felt on the undersurface of the diaphragm or liver. Partial omentectomy was done and the peritoneal biopsy was taken, same sent for histopathological examination. Postsurgery fetal cardiac activity was present. Her third postoperative day, USG revealed no FCA. The patient was discharged under a stable condition on the fourth postoperative day. Currently, the patient is on regular follow up in the infertility clinic.

\section{DISCUSSION}

Ovarian torsion is a partial or complete rotation of ovary around its vascular axis. ${ }^{6}$ In the early stages, only venous and lymphatic occlusion occurs, which results in enlargement of the ovary (sometimes massive). However, any delay in diagnosis or management will lead to arterial stasis resulting in hemorrhagic infarction and finally necrosis of the ovary..$^{5}$ Isolated torsion of the ovary is extremely rare. It generally involves both ovary and fallopian tube. There is two times higher risk of torsion on the right side as the mobility of the left ovary is limited by the sigmoid colon. ${ }^{7}$

Ovarian torsion after IVF/ IUI is extremely rare. The controlled ovarian stimulation during infertility treatment leads to development of the theca lutein cysts and an increase in ovarian size, predisposing to ovarian torsion.

Patients usually present with acute onset of severe, mostly unilateral lower abdominal pain which may be associated with nausea, vomiting. In advance cases, ischemic necrosis of the ovary will lead to an elevated temperature, increased heart rate, and hypotension. Only one-third of the patients present with tenderness on palpation, so its absence doesn't rule out torsion. ${ }^{5}$

The most important investigation for the diagnosis of torsion is ultrasound. ${ }^{8}$ Heterogeneously enlarged ovary in the midline, the presence of peripheral follicles, free fluid in the pouch of Douglas, twisted pedicle leading to whirlpool sign (The twisted vascular pedicle is visualized on ultrasound as an ellipsoid or tubular mass with internal heterogeneous echoes) or asymmetric enlargement of ovarian cyst wall indicate ovarian torsion on ultrasound. Presence of decreased perfusion of ovaries on color Doppler indicates torsion. Absent flow indicates a nonviable and necrotic ovary. ${ }^{9}$ However normal blood flow to ovaries does not rule out torsion. ${ }^{8}$ Once the diagnosis of ovarian torsion is confirmed, immediate surgical intervention is required to preserve ovarian function. ${ }^{10} \mathrm{In}$ the early stages, detorsion of the twisted pedicle can preserve ovarian function. However, in advance cases, when necrosis has already set in, adnexectomy needs to be done. In case oophorectomy is required, the patient should be given progesterone support until 12th weeks of gestation. $^{10}$

\section{CONCLUSION}

The decision of individualized and mild ovarian stimulation prior to IVF/IUI can markedly decrease the risk of ovarian torsion. Losing an ovary in an infertile female due to delay in diagnosis of ovarian torsion is absolutely unacceptable. So, if a pregnant patient presents with acute onset of severe abdominal pain, the diagnosis of ovarian torsion should always be ruled out. Ultrasound remains the mainstay for diagnosis of ovarian torsion. Normal blood flow on Doppler doesn't rule out torsion. In the early stages, detorsion of the twisted pedicle can help preserve the ovarian function. However, advance cases require adnexectomy.

Diagnosis of ovarian torsion requires a high index of clinical suspicion. Ultrasound with Doppler helps in diagnosing adnexal mass with torsion. Laparoscopy can help in both diagnosis and treatment with least morbidity. Conservative surgery should always be preferred to preserve fertility.

\section{ACKNOWLEDGMENTS}

Authors would like to thank the staff of Post Graduate Institute of Medical Education and Research, Chandigarh for great exposure.

\section{Funding: No funding sources \\ Conflict of interest: None declared \\ Ethical approval: Not required}

\section{REFERENCES}

1. Smith LP, Oskowitz SP, Barrett B, Bayer SR. IVF and embryo development subsequent to ovarian torsion occurring during the resumption of meiosis. Reprod Biomed Online. 2010;21(3):418-21.

2. Jungheim ES, Meyer MF, Broughton DE. Best practices for controlled ovarian stimulation in in vitro fertilization. Semin Reprod Med. 2015;33(2):77-82.

3. Spitzer D, Wirleitner B, Steiner H, Zech NH. Adnexal Torsion in Pregnancy after Assisted Reproduction - Case Study and Review of the Literature. Geburtshilfe Frauenheilkd. 2012;72(8):716-20.

4. Gil Navarro N, Garcia Grau E, Pina Perez S, Ribot Luna L. Ovarian torsion and spontaneous ovarian hyperstimulation syndrome in a twin pregnancy: A case report. Int J Surg Case Rep. 2017;34:66-8. 
5. Huang C, Hong MK, Ding DC. A review of ovary torsion. Ci Ji Yi Xue Za Zhi. 2017;29(3):143-7.

6. Chang HC, Bhatt S, Dogra VS. Pearls and pitfalls in diagnosis of ovarian torsion. Radiograph. 2008;28(5):1355-68.

7. Poonai N, Poonai C, Lim R, Lynch T. Pediatric ovarian torsion: case series and review of the literature. Can J Surg. 2013;56(2):103-8.

8. Rostamzadeh A, Mirfendereski S, Rezaie MJ, Rezaei S. Diagnostic efficacy of sonography for diagnosis of ovarian torsion. Pak J Med Sci. 2014;30(2):413-6.

9. Vijayaraghavan SB. Sonographic whirlpool sign in ovarian torsion. J Ultrasound Med. 2004;23(12):1643-9.
10. Ashwal E, Krissi H, Hiersch L, Less S, Eitan R, Peled Y. Presentation, Diagnosis, and Treatment of Ovarian Torsion in Premenarchal Girls. J Pediatr Adolesc Gynecol. 2015;28(6):526-9.

Cite this article as: Sachdeva G, Gainder S. Fetal Outcome of pregnancy following IVF/ IUI complicated by ovarian torsion: case reports. Int $\mathrm{J}$ Reprod Contracept Obstet Gynecol 2018;7:5169-72. 